\title{
THE IDENTITY IS THE MOST LIKELY EXCHANGE SHUFFLE FOR LARGE $n$
}

\author{
DANIEL GOLDSTEIN AND DAVID MOEWS
}

\begin{abstract}
Let a deck of $n$ cards be shuffled by successively exchanging the cards in positions $1,2, \ldots, n$ with cards in randomly chosen positions. We show that for $n \geq 18$, the identity permutation is the most likely. We prove a surprising symmetry of the resulting distribution on permutations. We also obtain the limiting distribution of the number of fixed points as $n \rightarrow \infty$.
\end{abstract}

\section{INTRODUCTION}

One way to shuffle a deck of $n$ cards is as follows. Start by numbering the positions of the cards from 1 to $n$. Swap the first card with a card in a randomly chosen position (possibly itself). Then swap the second card with a card in a randomly chosen position, and so on; the last exchange swaps the $n$th card with a card in a randomly chosen position. Robbins and Bolker [5] and Schmidt and Simion [7] have studied the probability distribution on the set of permutations of $\{1, \ldots, n\}$ induced by this exchange shuffle. In Section 2 we introduce the directed graph of an exchange shuffle. It has vertex set $\{1,2, \ldots, n\}$ and one edge for each swap. A first result relating the graph to the permutation says that a connected component of the graph corresponds to either a cycle or a product of two disjoint cycles. In the first case we say that the graph is a tree; in the second case we say that the graph is a unicycle because it contains a unique cycle. This result is used in Section 3 to show that for large $n$ the identity is the most frequent permutation. In Section 4, we present a second result based on a more detailed study of the graph. For a unicyclic graph, this result says which vertices lie in which cycle, and gives a formula for the probability of the permutation. This will be used in Sections 5 and 6 . In Section 5 we prove various facts about the probability of the concatenation of a series of permutations. In Section 6, we find the most likely permutation in each of the conjugacy classes of $S_{n}$. We prove that for $n \geq 18$, the identity is most frequent, and for $4 \leq n \leq 17$ the most frequent permutation is $(n \cdots m+1)(m \cdots 1)$, where $m$ is $n / 2$ if $n$ is even, and either $(n-1) / 2$ or $(n+1) / 2$ if $n$ is odd. This answers a question of Robbins and Bolker. In Section 7 we determine the limiting distribution as $n \rightarrow \infty$ of the number of fixed points generated by the exchange shuffle (and a related shuffle), thus answering a question of Schmidt and Simion.

Date: June 1, 2018.

1991 Mathematics Subject Classification. 05A15. 


\section{The GRAPH OF A SHUfFLE}

Suppose $\pi$ is a permutation of the set $\{1, \ldots, n\}$. We wish to count the number of ways of representing $\pi$ as a product of transpositions

$$
\pi=\left(n a_{n}\right) \cdots\left(2 a_{2}\right)\left(1 a_{1}\right), \quad a_{1}, \ldots, a_{n} \in\{1, \ldots, n\} .
$$

Call this number the multiplicity $N(\pi)$ of $\pi$. As the total number of exchange shuffles is $n^{n}$, the probability that the permutation $\pi$ arises as an exchange shuffle is $N(\pi) / n^{n}$. In this paper we shall usually consider multiplicities and not probabilities.

Consider a permutation $\pi$ and its representation (11) as a product of transpositions. We can construct a (possibly loop-containing) directed graph (digraph) from this representation by taking $\{1, \ldots, n\}$ to be the set of vertices, and directing an edge from $j$ to $a_{j}$ for each $j$. We will say that this digraph also represents $\pi$.

Each vertex has out-degree one, so every connected component of the graph contains a unique directed cycle, possibly with just one vertex. For, suppose we are given a connected component with vertex set of cardinality $m$. Since each vertex has out-degree one, the component has exactly $m$ edges; hence there is at most one cycle, directed or otherwise. Choose a vertex $v_{1}$ and form a sequence inductively by setting $v_{i+1}=a_{v_{i}}$. By the pigeon-hole principle this sequence repeats. This repeating part is the unique directed cycle.

To avoid confusion with cycles of permutations, we will call this directed cycle a ring. The component can also contain trees rooted on this cycle with edges directed towards the ring. The transpositions in different connected components of the digraph do not interact, so our exchange shuffle induces a permutation on the vertex set of each component of the digraph.

Proposition 1. [7, Lemmas 1.2, 1.4] If the ring has length 1, the permutation $\rho$ induced on a connected component of the digraph with $m$ vertices is an $m$-cycle. If the ring has length 2 or greater, $\rho$ is a product of two disjoint cycles whose lengths sum to $\mathrm{m}$. In the first case we call the component a tree, in the second a unicycle.

Proof. Suppose the ring has length 1. Then the component is a rooted tree with a loop at the root. The loop has no effect on the permutation generated. Ignore it, and suppress the corresponding transposition. This leaves us with a permutation of the form

$$
\rho=\left(\begin{array}{ll}
b_{m-1} & c_{m-1}
\end{array}\right)\left(b_{m-2} c_{m-2}\right) \cdots\left(b_{1} c_{1}\right),
$$

whose associated graph is a tree $\mathcal{T}$ with vertex set, $\mathcal{S}$ say, of cardinality $m$, and with edge set $\left\{\left(b_{i}, c_{i}\right) \mid 1 \leq i \leq m-1\right\}$.

We wish to prove by induction on $m$ that (2) is an $m$-cycle. For $m=1$ this is true. For $m$ larger, let $e=\left(b_{i}, c_{i}\right)$ be any edge of $\mathcal{T}$ with $b_{i}$ a leaf vertex. Deleting $e$ and $b_{i}$ leaves a tree on the vertex set $\mathcal{S} \backslash\left\{b_{i}\right\}$ of cardinality $m-1$, so, by the induction hypothesis, the permutation

$$
\rho^{\prime}=\left(\begin{array}{lll}
b_{i-1} & c_{i-1}
\end{array}\right) \cdots\left(b_{1} c_{1}\right)\left(b_{m} c_{m}\right) \cdots\left(b_{i+1} c_{i+1}\right)
$$

is a cycle. Write

$$
\rho^{\prime}=\left(c_{i} h_{1} \cdots h_{m-2}\right)
$$

Then $\rho$ is conjugate to

$$
\left(b_{i} c_{i}\right) \rho^{\prime}=\left(\begin{array}{llll}
c_{i} & h_{1} & \cdots & h_{m-2} \\
b_{i}
\end{array}\right) .
$$


However, a conjugate of a cycle is a cycle. This completes the induction.

Next, we treat the case of a ring of length 2 or greater. Let

$$
\rho=\left(f_{m} g_{m}\right) \cdots\left(f_{2} g_{2}\right)\left(f_{1} g_{1}\right) .
$$

Choose an edge $\left(f_{i}, g_{i}\right)$ of the ring. Deleting it changes our graph component into a tree, so

$$
\rho^{\prime}=\left(f_{i-1} g_{i-1}\right) \cdots\left(f_{2} g_{2}\right)\left(f_{1} g_{1}\right)\left(f_{m} g_{m}\right) \cdots\left(f_{i+1} g_{i+1}\right)
$$

is of the form (2). Then, as we just proved, $\rho^{\prime}$ is an $m$-cycle. However, a product of an $m$-cycle and a transposition of two of its members will equal a product of two disjoint cycles whose lengths sum to $m$, thus proving the lemma. In fact, if we write

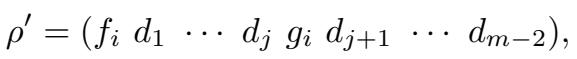

$\rho$ will be conjugate to

$$
\left(f_{i} g_{i}\right) \rho^{\prime}=\left(f_{i} d_{1} \cdots d_{j}\right)\left(g_{i} d_{j+1} \cdots d_{m-2}\right),
$$

a product of two disjoint cycles whose lengths sum to $m$. But conjugation does not change cycle structure, so $\rho$ also has this form.

We can define the multiplicity $N(\pi)$ of a permutation of any set of nonnegative integers just as we defined the multiplicity of permutations of $\{1, \ldots, n\}$. Write $N^{\text {tree }}(\pi)$ for the number of representations of $\pi$ as a tree, or tree multiplicity of $\pi$, and $N^{\text {uni }}(\pi)$ for the number of representations of $\pi$ as a unicycle, or unicyclic multiplicity of $\pi$. Obviously, $N(\pi), N^{\text {tree }}(\pi)$, and $N^{\text {uni }}(\pi)$ are invariant under conjugation of $\pi$ by an order-preserving map.

Lemma 2. For a permutation of $\{1, \ldots, n\}$ that is a product $\pi=\pi_{1} \pi_{2} \cdots \pi_{q}$ of $q$ disjoint cycles, we have

$$
N(\pi)=\sum_{\chi \text { an involution of }} \prod_{\chi 1, \ldots, q\}} N^{\text {tree }}\left(\pi_{i}\right) \prod_{\chi(i)=j, i<j} N^{\mathrm{uni}}\left(\pi_{i} \pi_{j}\right) .
$$

Proof. Any representation of $\pi$ as an exchange shuffle will determine a partition of the set $\left\{\pi_{1}, \ldots, \pi_{q}\right\}$ into one- and two-element blocks, the one-element blocks being those cycles represented as trees, and the two-element blocks those pairs of cycles represented as unicycles. Think of such a partition as an involution, by letting an involution $\chi$ partition $\left\{\pi_{1}, \ldots, \pi_{q}\right\}$ into its orbits under $\chi$. The product of $N^{\text {tree }}\left(\pi_{i}\right)$ over the fixed points $\pi_{i}$ of $\chi$ multiplied by the product of $N^{\text {uni }}\left(\pi_{j} \pi_{k}\right)$ over the two-element orbits $\left\{\pi_{j}, \pi_{k}\right\}$ of $\chi$ will then equal the number of representations of $\pi$ yielding this $\chi$, and summing this over all involutions $\chi$ will yield $N(\pi)$.

\section{The IDENTITy IS MOST FREQUENT FOR LARGE $n$}

Using Lemma 2 and the following three lemmas will enable us to prove that the identity permutation has highest multiplicity for $n \geq 29$. In Section 6 we will obtain a stronger result.

Lemma 3. Let $Q_{n}$ be the number of involutions on an n-element set. Then

1. $Q_{n} 4^{-n}$ is strictly increasing for $n \geq 15$,

2. $Q_{n} 4^{-n} \leq \frac{1}{4}$ for $n<15$, and

3. $Q_{29} 4^{-29}>\frac{1}{4}$. 
Proof. We have

$$
Q_{0}=Q_{1}=1 .
$$

For $n \geq 2$, an involution on $\{1, \ldots, n\}$ can either fix the first element, leaving $Q_{n-1}$ ways of moving the remaining elements, or swap it with a member of $\{2, \ldots, n\}$, leaving $Q_{n-2}$ ways of moving the remaining elements. Hence

$$
Q_{n}=Q_{n-1}+(n-1) Q_{n-2}, \quad \text { for } n \geq 2 .
$$

If we set

$$
R_{n}=\frac{Q_{n}}{Q_{n-1}}
$$

then induction on $n$ proves that, for all $n \geq 2$,

$$
\sqrt{n}<R_{n}<\sqrt{n}+1 .
$$

From the above, $Q_{n}>\sqrt{n} Q_{n-1}$, so $Q_{n} 4^{-n}$ will be strictly increasing for $n \geq 15$. Inequalities (2) and (3) require a calculation, which we omit.

Let $N^{\text {tree, } x}(\pi)$ be the number of representations of $\pi$ as a tree where we constrain $x$ to be the root of the tree, i.e., we constrain the transposition $(x x)$ to be made. Let $C_{n}=\frac{1}{n+1}\left(\begin{array}{c}2 n \\ n\end{array}\right)$ be the $n$th Catalan number.

Lemma 4. [5, Theorems 8 and 9] For cycles $\mu$ on an n-element set, $N^{\text {tree }}(\mu)=$ $N(\mu)$ has maximum value $C_{n}$. Let $\mu_{1}$ be the smallest element of $\mu$; then $N^{\text {tree, } \mu_{1}}(\mu)$ has maximum value $C_{n-1}$. Both these maxima are achieved only when $\mu$ can be written as $\left(\mu_{n} \mu_{n-1} \cdots \mu_{1}\right)$, where $\mu_{1}<\mu_{2}<\cdots<\mu_{n}$.

Proof. See Robbins and Bolker.

Lemma 5. If $\pi$ acts on an n-element set, then $N^{\text {tree }}(\pi) \leq 4^{n-1}$ for $n \geq 1$, and $N^{\text {uni }}(\pi) \leq 4^{n-2}$ for $n \geq 2$.

Proof. We have just seen that $N^{\text {tree }}(\pi) \leq C_{n}$. Since $C_{1}=1$, the first inequality is true for $n=1$. We have the following form of Stirling's formula [6]:

$$
\sqrt{2 \pi m}(m / e)^{m} e^{1 /(12 m+1)} \leq m ! \leq \sqrt{2 \pi m}(m / e)^{m} e^{1 / 12 m}, \quad m \geq 1 .
$$

For $n \geq 2$, this yields the estimate $C_{n} \leq 4^{n} /(n+1) \sqrt{\pi n}$, which implies the result.

For the second inequality, let $W_{n}$ be the maximum of $N^{\text {uni }}(\pi)$ over all permutations $\pi$ of $\{1, \ldots, n\}$. We will induce on $n$ to prove that $W_{n} \leq 4^{n-2}$ for all $n \geq 2$. For $N^{\text {uni }}(\pi)$ to be nonzero, $\pi$ must be a product of two disjoint cycles, $\left(b_{1} b_{2} \cdots b_{m}\right)\left(b_{m+1} \cdots b_{n}\right)$, say. Without loss of generality, let $b_{m}=1$. Represent $\pi$ in the form (11), and let the first transposition be $\left(1 b_{i}\right), i \neq m$. The remaining transpositions must represent $\pi\left(1 b_{i}\right)$, and they will still do so if we precede them by (1 1). If $i>m, \pi\left(1 b_{i}\right)$ is an $n$-cycle, so the representation is as a tree, and the tree has a loop at 1 . We thus get at most $N^{\text {tree, } 1}\left(\pi\left(1 b_{i}\right)\right)$ choices for the remaining transpositions; by Lemma 4 , this is no more than $C_{n-1}$. If $1 \leq i \leq m-1$,

$$
\pi\left(1 b_{i}\right)=\left(\begin{array}{llll}
b_{1} & b_{2} & \cdots & b_{i}
\end{array}\right)\left(b_{i+1} b_{i+2} \cdots b_{m}\right)\left(b_{m+1} \cdots b_{n}\right) .
$$

Our representation of $\pi\left(1 b_{i}\right)$ has a loop at 1 , so it must contain a tree component rooted at 1 representing $\left(b_{i+1} \cdots b_{m}=1\right)$; for the representation of $\pi$ to be unicyclic, it must also contain a unicyclic component representing $\left(b_{1} b_{2} \cdots b_{i}\right)\left(b_{m+1} \cdots b_{n}\right)$, 
so by Lemma 1 , the number of ways of filling in the remaining transpositions is then no more than $C_{m-i-1} W_{n-m+i}$. Then

$$
N^{\mathrm{uni}}(\pi) \leq(n-m) C_{n-1}+\sum_{1 \leq i \leq m-1} C_{m-i-1} W_{n-m+i}
$$

and since for all possible $\pi, 1 \leq m \leq n-1$,

$$
W_{n} \leq(n-1) C_{n-1}+\sum_{0 \leq j \leq n-3} C_{j} W_{n-j-1} .
$$

$W_{n} \leq 4^{n-2}$ for $2 \leq n \leq 9$ can be checked via direct computer enumeration (or using some of our results below), and using the bound (3) recursively then yields $W_{n} \leq 4^{n-2}$ for $10 \leq n \leq 21$; so let $n \geq 22$. Then by induction,

$$
\begin{aligned}
W_{n} & \leq(n-1) C_{n-1}+\sum_{0 \leq j \leq n-3} C_{j} 4^{n-j-3} \\
& \leq(n-1) C_{n-1}+4^{n-3} \sum_{j \geq 0} C_{j} 4^{-j} .
\end{aligned}
$$

Substitution into the generating function for $C_{n}$ yields $\sum_{j \geq 0} C_{j} 4^{-j}=2$, and estimation with Stirling's formula as above yields $(n-1) C_{n-1} \leq 2 \cdot 4^{n-3}$ for $n-1 \geq 64 / \pi$, i.e., $n \geq 22$. This completes the proof.

Proposition 6. For $n \geq 29$, the identity has larger multiplicity than any other permutation of $\{1,2, \ldots, n\}$.

Proof. Let $\pi$ be an arbitrary permutation of $\{1, \ldots, n\}$, and let $\pi=\pi_{1} \cdots \pi_{q}$ be its decomposition into disjoint cycles. By Lemma 2 ,

$$
N(\pi)=\sum_{\chi \text { an involution of }} \prod_{\chi(1, \ldots, q\}} N^{\text {tree }}\left(\pi_{i}\right) \prod_{\chi(i)=j, i<j} N^{\text {uni }}\left(\pi_{i} \pi_{j}\right) .
$$

Plugging in the bounds from Lemma above,

i.e.,

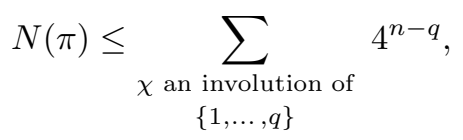

$$
N(\pi) \leq Q_{q} 4^{n-q} .
$$

From Lemma 3, it follows that for $n \geq 29, Q_{q} 4^{-q}$ is maximized at $q=n$, so $N(\pi)<Q_{n}$ unless $q=n$, i.e., unless $\pi$ is the identity permutation. However the multiplicity of the identity permutation is $Q_{n}$, by [ [5, Theorem 6], or directly from Lemma 2. This completes the proof.

\section{A RESUlt ON UNICYCLIC MULTIPLICITY}

The main result of this section, Proposition 8, is a formula for the multiplicity of a product of two disjoint cycles.

Return to Proposition 11. In the case that the connected component is a unicycle, the permutation is a product of two disjoint cycles, and we will now say which ring vertices are in which cycle. If a ring vertex is larger than its predecessor, it's in one cycle. If it's smaller, it's in the other. This is illustrated in Figure 1. 

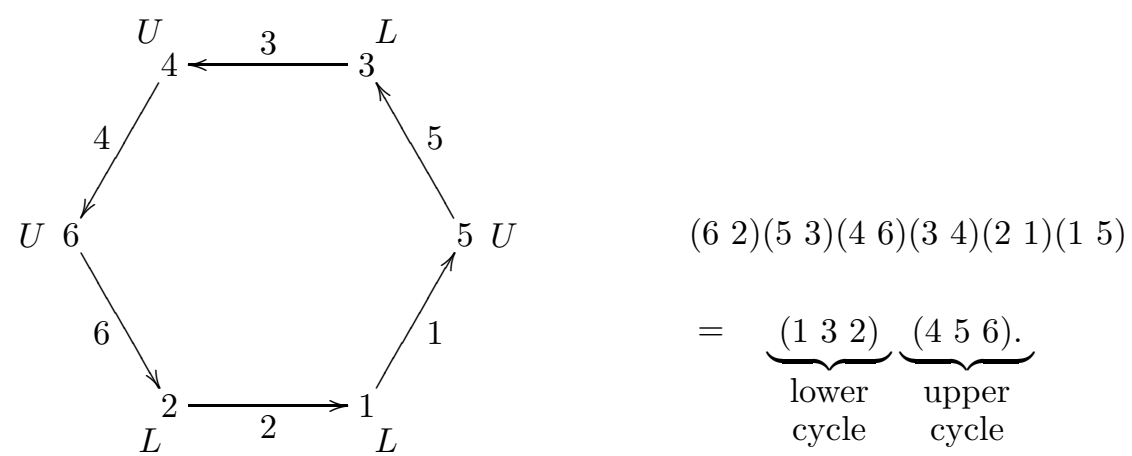

Figure 1. The lower and upper cycle of a unicyclic component.

Proposition 7. [7, Lemma 1.4] The permutation induced on a digraph component which is a tree is a cycle, and the permutation induced on a digraph component which is a unicycle is a product of two disjoint cycles. For a unicyclic component, divide the vertices in the ring into upper vertices, whose predecessors in the ring have a smaller label, and lower vertices, whose predecessors have a larger label. Then one cycle, the upper cycle, contains all the upper vertices, and the other cycle, the lower cycle, contains all the lower vertices.

Remark 1. We will find it useful from now on to label edges in our digraphs by their source vertices. This was already seen in Figure 1.

Remark 2. Later, in Proposition 8, we will see which non-ring vertices are upper and which are lower. We will also see what order to take them in.

Proof. First, let the component be a tree. We will prove the induced permutation is a cycle, but now without using conjugation. As before, let us suppose we have a permutation of the form (5):

$$
\begin{aligned}
\rho= & \left(b_{m-1} c_{m-1}\right)\left(b_{m-2} c_{m-2}\right) \cdots\left(b_{1} c_{1}\right), \\
& \text { whose associated graph, } \mathcal{T}, \text { is a tree with vertex set } \mathcal{S} \text { of } \\
& \text { cardinality } m \text { and edge set }\left\{\left(b_{1}, c_{1}\right), \ldots,\left(b_{m-1}, c_{m-1}\right)\right\} .
\end{aligned}
$$

We wish to prove by induction on $m$ that (5) is an $m$-cycle. We will need a stronger induction hypothesis. Let $b_{1}, \ldots, b_{m-1}$ be thought of as an increasing sequence of times of day, and let a postman travel on the set $\mathcal{S}$. If he is at $b_{i}$ at time $b_{i}$, he will move to $c_{i}$, or, if he is at $c_{i}$ at time $b_{i}$, he will move to $b_{i}$. Otherwise, he will stay fixed. Our induction hypothesis is that, regardless of where and at what time of day the postman starts, he will return to the same place at the same time of day $m$ days later, and that his intervening journey will, for any time of day $t$ and $s \in \mathcal{S}$, find him at $s$ at time $t$ exactly once.

For $m=1$ this is clear, since the postman simply stays put for one day. Otherwise, let $e=\left(b_{i}, c_{i}\right)$ be any edge of $\mathcal{T}$. Deleting $e$ will split $\mathcal{T}$ into subtrees; let the subtree where the postman starts his journey be $\mathcal{T}^{\prime}$, with $m^{\prime}$ vertices, say, and let the other subtree be $\mathcal{T}^{\prime \prime}$, with $m^{\prime \prime}=m-m^{\prime}$ vertices. By the induction hypothesis on $\mathcal{T}^{\prime}$, the postman will travel until he reaches an endpoint of $e$ at time of day $b_{i}$, when he will travel to the other endpoint of $e$ in $\mathcal{T}^{\prime \prime}$. Then, by the induction hypothesis on $\mathcal{T}^{\prime \prime}$, he will find himself at the endpoint of $e$ at time of day $b_{i}$ exactly 
$m^{\prime \prime}$ days later, when he will travel back to $\mathcal{T}^{\prime}$. His journey there will then go on as if he had never left, so he will arrive back at his starting point and starting time of day after having spent $m^{\prime}$ days in $\mathcal{T}^{\prime}$ and $m^{\prime \prime}$ days in $\mathcal{T}^{\prime \prime}$, for a total of $m^{\prime}+m^{\prime \prime}=m$ days. In fact, his cyclical journey in $\mathcal{T}$ simply consists of his journeys in $\mathcal{T}^{\prime}$ and $\mathcal{T}^{\prime \prime}$ spliced together, so it's clear that the induction hypothesis is still satisfied.

If we let the postman be at $v$ at the start of the day (before any time $b_{i}$ ), then he will be at $\rho(v)$ at the end of the day (after all times $b_{i}$.) We may say that he stops for the night there. At any rate, he is still at $\rho(v)$ at the start of the next day; he is at $\rho^{2}(v)$ at the start of the day after that; and so on. Our induction hypothesis then tells us that $v, \rho(v), \ldots, \rho^{m-1}(v)$ are distinct, so $\rho$ is an $m$-cycle, as desired.

If the component is unicyclic, suppose at first that there are no edges other than those in the ring, and draw the ring in the plane so that it is oriented counterclockwise. If the postman spends the night at an upper vertex, he will leave via the incoming edge, in a clockwise direction, and continue to travel clockwise, passing through all the lower vertices he meets on the way, before spending the next night at the next upper vertex. Therefore, the upper vertices form a cycle in the induced permutation. Similarly, if he spends a night at a lower vertex, the postman will spend his next night at the next lower vertex in a counterclockwise direction, so the lower vertices also form a cycle. We illustrate this in Figure 1. In the general case, the unicyclic component will contain trees attached to the ring by edges. However, we already know that each such tree will, on its own, generate a cyclical journey. When attached to the ring, these journeys will be spliced into the journeys around the cycles of upper and lower vertices, as explained above in the proof that the induced permutation on a tree is a cycle. This completes the proof.

We wish to state a formula for $N^{\text {uni }}$. Let $\pi$ be a permutation on a set of nonnegative integers that contains zero. Write $N^{\prime}(\pi)$ for $N^{\text {tree, } 0}(\pi)$, the number of representations of $\pi$ as a tree with root 0 . Given a tree $T$ with root 0 , let $\beta_{+}(T)$ be the largest vertex adjacent to 0 , and $\beta_{-}(T)$ the smallest. Then we write $N_{\beta_{+}>x}^{\prime}(\pi)$ for the number of representations of $\pi$ as a tree $T$ with root 0 and $\beta_{+}(T)>x$; $N_{\beta_{-}<x}^{\prime}(\pi)$ is defined similarly.

We will use capital letters $A, B, D$, and $E$ to denote nonempty sequences of distinct nonnegative integers; the concatenation of sequences $A$ and $B$ will be written $A B$. Let $\gamma_{-}(A)$ be the first element of $A$, and $\gamma_{+}(A)$ the last. Let $N_{u}^{\text {uni }}((A),(B))$ be the number of representations of the permutation $(A)(B)$ as a unicyclic digraph for which $(A)$ is the upper cycle. We can now state our formula for $N^{\text {uni }}$.

Proposition 8. For all disjoint sequences of distinct positive integers $A$ and $B$, $N_{u}^{\text {uni }}((A),(B))$ is equal to

$$
\sum_{k \geq 1} \frac{1}{k} \sum_{\begin{array}{c}
\left(A_{k} \cdots A_{1}\right)=(A) \\
\left(B_{1} \cdots B_{k}\right)=(B)
\end{array}} \prod_{i} N_{\beta_{+}>\gamma_{-}\left(B_{i}\right)}^{\prime}\left(\left(A_{i} 0\right)\right) \prod_{j} N_{\beta_{-}<\gamma_{+}\left(A_{j+1}\right)}^{\prime}\left(\left(B_{j} 0\right)\right)
$$

where we take $A_{k+1}=A_{1}$.

Proof. First we comment on what is to be proved. Start with the left-hand side above. Suppose we are given a representation of $(A)(B)$ as a unicyclic exchange shuffle, and suppose that in the associated directed graph $(A)$ is the upper and $(B)$ the lower cycle. Break up the ring into maximal segments of consecutive vertices 
that are all upper or all lower. Say there are $2 k$ such segments. Beginning with an arbitrary upper segment, write the vertices of the ring as

$$
\text { Ring }=D_{1} E_{1} D_{2} E_{2} \cdots D_{k} E_{k} \text {. }
$$

Of course, there are $k$ ways of doing this, depending on which upper segment we begin with.

We construct a forest of $k$ trees by, for each $i=1, \ldots, k$, doing the following:

1. Start with the digraph representing $(A)(B)$.

2. Follow the postman as he traverses the part $D_{i}$ of the ring, and certain trees hanging off the ring (see Figure 2).

3. Use the dummy vertex 0 for any ring vertices the postman traverses that are not in $D_{i}$.

In this way, we get upper trees $T_{i}$ for $1 \leq i \leq k$. By a similar process, to be described below, we get lower trees $U_{j}$ for $1 \leq j \leq k$. We get corresponding decompositions

$$
A=A_{k} A_{k-1} \cdots A_{1}
$$

and

$$
B=B_{1} B_{2} \cdots B_{k} .
$$

The details of these decompositions are illustrated in Figures 2 and 3 . These $2 k$ trees and $2 k$ sequences satisfy the properties implied by the right-hand side above:

1. The tree $T_{i}$ represents $\left(A_{i} 0\right)$. The tree $U_{j}$ represents $\left(B_{j} 0\right)$.

2. The largest neighbor of 0 in the tree $T_{i}$ is greater than the first element of $B_{i}$. The smallest neighbor of 0 in the tree $U_{j}$ is less than the last element of $A_{j+1}$.

We let these indices wrap around modulo $k$, so that $A_{k+1}=A_{1}$.

The content of Theorem 8 is that this decomposition exists, and, conversely, that any choice of positive integer $k$ and choice of $2 k$ subsequences and $2 k$ tree representations thereof satisfying conditions 1 and 2 above give rise to a unicyclic representation of $(A)(B)$ where $(A)$ is the upper and $(B)$ the lower cycle.

Next we proceed to the proof. Consider a representation of $(A)(B)$ as a unicycle, where $(A)$ is the upper cycle. Take the vertices of the ring to be, in order, $\alpha_{1}$, $\alpha_{2}, \ldots, \alpha_{m}$, where $\alpha_{1}$ is upper and $\alpha_{m}$ lower. We will let these indices wrap around modulo $m$, so $\alpha_{0}=\alpha_{m}, \alpha_{m+1}=\alpha_{1}$, and so on. Start with a maximal consecutive subsequence $D_{i}=\alpha_{a} \alpha_{a+1} \cdots \alpha_{a+b}$ of upper vertices among the $\alpha_{i}$ 's, and let $\alpha_{a+b+1} \cdots \alpha_{a+b+c}$ be the following maximal consecutive subsequence of lower vertices. Suppose that our postman has just started out for the day from $\alpha_{a+b+c+1}$, which is an upper vertex, by maximality. What happens to him in the remainder of his journey?

Since $\alpha_{a+b+c+1}$ is upper, $\alpha_{a+b+c+1}>\alpha_{a+b+c}$, so he will eventually be leaving along the edge labeled $\alpha_{a+b+c}$; but first, he may traverse subtrees of the unicyclic component attached to the ring at $\alpha_{a+b+c+1}$. Call a collection of subtrees attached to the ring by edges labeled $j$, where $\kappa<j<\lambda$, a $\mathcal{T}(\kappa, \lambda)$. We can now say that our postman will traverse the $\mathcal{T}\left(-\infty, \alpha_{a+b+c}\right)$ incident on $\alpha_{a+b+c+1}$. Following this, he arrives at $\alpha_{a+b+c}$. This is a lower vertex, so $\alpha_{a+b+c-1}>\alpha_{a+b+c}$. He will therefore traverse the incident $\mathcal{T}\left(\alpha_{a+b+c}, \alpha_{a+b+c-1}\right)$ and continue to $\alpha_{a+b+c-1}$ without stopping for the night. If this vertex is a lower vertex, he will traverse $\mathcal{T}\left(\alpha_{a+b+c-1}, \alpha_{a+b+c-2}\right)$ and continue to $\alpha_{a+b+c-2}$, and so on. Eventually, after 
traversing $\mathcal{T}\left(\alpha_{a+b+1}, \alpha_{a+b}\right)$, he will come to $\alpha_{a+b}$. This is an upper vertex, so $\alpha_{a+b-1}<\alpha_{a+b}$, meaning that he will traverse the incident $\mathcal{T}\left(\alpha_{a+b}, \infty\right)$, and then stop for the night at $\alpha_{a+b}$. The next day, he will traverse the $\mathcal{T}\left(-\infty, \alpha_{a+b-1}\right)$ before traveling to $\alpha_{a+b-1}$. Here he will traverse $\mathcal{T}\left(\alpha_{a+b-1}, \infty\right)$, stop at $\alpha_{a+b-1}$, and traverse $\mathcal{T}\left(-\infty, \alpha_{a+b-2}\right)$ before passing to $\alpha_{a+b-2}$, and he will continue in this way until reaching $\alpha_{a}$ and stopping for the night. Since we are now in the same position we started out in (although with different values of $a, b$, and $c$ ), we end our description here. This travelogue generates a sequence which forms a portion of the cycle $(A)$. Equating our $\mathcal{T}(\kappa, \lambda)$ 's with the sequence of vertices they generate when traversed, we may write it as

$$
\begin{aligned}
(7) A_{i}= & \left.\mathcal{T}\left(-\infty, \alpha_{a+b+c}\right) \quad \text { (rooted at } \alpha_{a+b+c+1}\right) \\
& \mathcal{T}\left(\alpha_{a+b+c}, \alpha_{a+b+c-1}\right) \quad\left(\text { rooted at } \alpha_{a+b+c}\right) \\
& \vdots \\
& \mathcal{T}\left(\alpha_{a+b+1}, \alpha_{a+b}\right) \quad\left(\text { rooted at } \alpha_{a+b+1}\right) \\
& \left.\mathcal{T}\left(\alpha_{a+b}, \infty\right) \alpha_{a+b} \mathcal{T}\left(-\infty, \alpha_{a+b-1}\right) \quad \text { (both trees rooted at } \alpha_{a+b}\right) \\
& \vdots \\
& \mathcal{T}\left(\alpha_{a+1}, \infty\right) \alpha_{a+1} \mathcal{T}\left(-\infty, \alpha_{a}\right) \quad\left(\text { both trees rooted at } \alpha_{a+1}\right) \\
& \mathcal{T}\left(\alpha_{a}, \infty\right) \alpha_{a} \quad\left(\text { tree rooted at } \alpha_{a}\right) .
\end{aligned}
$$

Generate a tree $T_{i}$ by the following procedure: take the vertices $\alpha_{a}, \alpha_{a+1}, \ldots$, $\alpha_{a+b}$, and the edges connecting them; add to this all the $\mathcal{T}(\kappa, \lambda)$ 's above rooted on these vertices. Add an edge from $\alpha_{a+b}$ to 0 , and take all the trees $\mathcal{T}\left(-\infty, \alpha_{a+b+c}\right)$, $\mathcal{T}\left(\alpha_{a+b+c}, \alpha_{a+b+c-1}\right), \ldots, \mathcal{T}\left(\alpha_{a+b+1}, \alpha_{a+b}\right)$ appearing above and reroot them at 0. Walking the postman through $T_{i}$ tells us immediately that it is a representation of $\left(A_{i} 0\right)$. Observe that $\beta_{+}\left(T_{i}\right)=\alpha_{a+b}$, and that $\gamma_{+}\left(A_{i}\right)=\alpha_{a}$. All this is illustrated in Figure 2.

Let us interchange 'lower' and 'upper' above, so that our starting subsequence is a maximal consecutive subsequence $E_{j}=\alpha_{d} \alpha_{d+1} \cdots \alpha_{d+e}$ of lower vertices, $\alpha_{d+e+1}, \ldots, \alpha_{d+e+f}$ are upper, and $\alpha_{d+e+f+1}$ is lower. Supposing that our postman is just about to stop for the night at $\alpha_{d}$, he will travel along the sequence

$$
\begin{aligned}
B_{j}= & \alpha_{d} \mathcal{T}\left(-\infty, \alpha_{d}\right) \quad\left(\text { tree rooted at } \alpha_{d}\right) \\
& \mathcal{T}\left(\alpha_{d}, \infty\right) \alpha_{d+1} \mathcal{T}\left(-\infty, \alpha_{d+1}\right) \quad\left(\text { both trees rooted at } \alpha_{d+1}\right) \\
& \vdots \\
& \left.\mathcal{T}\left(\alpha_{d+e-1}, \infty\right) \alpha_{d+e} \mathcal{T}\left(-\infty, \alpha_{d+e}\right) \quad \text { (both trees rooted at } \alpha_{d+e}\right) \\
& \mathcal{T}\left(\alpha_{d+e}, \alpha_{d+e+1}\right) \quad\left(\text { rooted at } \alpha_{d+e+1}\right) \\
& \vdots \\
& \mathcal{T}\left(\alpha_{d+e+f-1}, \alpha_{d+e+f}\right) \quad\left(\text { rooted at } \alpha_{d+e+f}\right) \\
& \mathcal{T}\left(\alpha_{d+e+f}, \infty\right) \quad\left(\text { rooted at } \alpha_{d+e+f+1}\right)
\end{aligned}
$$

before stopping for the night at $\alpha_{d+e+f+1}$, where we will leave him. As before, we may view $B_{j}$ as being part of $(B)$. Generate a tree $U_{j}$ by taking the vertices $\alpha_{d}, \alpha_{d+1}, \ldots, \alpha_{d+e}$, and the edges connecting them; add to this all the $\mathcal{T}(\kappa, \lambda)$ 's above rooted on these vertices. Add an edge from $\alpha_{d+e}$ to 0 , and take all the trees 
$\mathcal{T}\left(\alpha_{d+e}, \alpha_{d+e+1}\right), \ldots, \mathcal{T}\left(\alpha_{d+e+f-1}, \alpha_{d+e+f}\right), \mathcal{T}\left(\alpha_{d+e+f}, \infty\right)$ appearing above and reroot them at 0 . Walking the postman through $U_{j}$ tells us that it is a representation of $\left(B_{j} 0\right)$. In this case, $\beta_{-}\left(U_{j}\right)=\alpha_{d+e}$, and $\gamma_{-}\left(B_{j}\right)=\alpha_{d}$. This is illustrated in Figure 3.

Split the vertices occurring in the ring into blocks of upper and lower vertices, so that

$$
\alpha_{1} \cdots \alpha_{m}=D_{1} E_{1} D_{2} E_{2} \cdots D_{k} E_{k}
$$

for some sequences $D_{1}, \ldots, D_{k}$ of upper and $E_{1}, \ldots, E_{k}$ of lower vertices. We can then apply the above transformations to generate $A_{1}, \ldots, A_{k}, B_{1}, \ldots, B_{k}$ and associated tree representations $T_{i}$ of the $\left(A_{i} 0\right)$ 's and $U_{j}$ of the $\left(B_{j} 0\right)$ 's. Looking at the postman's journey tells us that $(A)=\left(A_{k} \cdots A_{1}\right)$ and $(B)=\left(B_{1} \cdots B_{k}\right)$. As above, let the first vertex of $D_{i}$ be $\alpha_{a}=\gamma_{+}\left(A_{i}\right)$. This must be upper, so $\alpha_{a-1}<\alpha_{a}$; but $\alpha_{a-1}$ is the last vertex of $E_{i-1}$, which is $\beta_{-}\left(U_{i-1}\right)$ (letting indices wrap around modulo $k)$, so $\beta_{-}\left(U_{i-1}\right)<\gamma_{+}\left(A_{i}\right)$. Similarly, $\beta_{+}\left(T_{i}\right)>\gamma_{-}\left(B_{i}\right)$.

In this way, we get a split of $(A)$ and $(B)$ into subsequences, and tree representations thereof, of exactly the kind counted on the right-hand side of (6). In fact, for each unicyclic representation whose ring contains $k$ blocks of upper vertices interleaved with $k$ blocks of lower vertices, we get $k$ such splits and representations, since we had $k$ choices for $\alpha_{1}$. This gives a map from the set of things counted on the left-hand side of (6) to the set of things counted on the right-hand side of (6).

To find an inverse map, we need to be able to splice a number of tree representations together into a unicycle. Suppose that we start with a tree $T_{i}$ which represents $\left(A_{i} 0\right)$. Our first task is to construct the corresponding $D_{i}=\alpha_{a} \cdots \alpha_{a+b}$, say. We may let $\alpha_{a+b}$ be the largest vertex with an edge to 0 . Set $\alpha_{a+b-1}$ to be the largest vertex less than $\alpha_{a+b}$ with an edge to $\alpha_{a+b}$, set $\alpha_{a+b-2}$ to the largest vertex less than $\alpha_{a+b-1}$ with an edge to $\alpha_{a+b-1}$, and so on, until we reach a vertex $\alpha_{a}$ such that no $x<\alpha_{a}$ has an edge to $\alpha_{a}$. The sequence of vertices constructed in this way obviously has $\alpha_{a}<\alpha_{a+1}<\cdots<\alpha_{a+b}$. The tree $T_{i}$ will consist of the path of edges from $\alpha_{a}$ to 0 , together with a $\mathcal{T}\left(\alpha_{a}, \infty\right)$ rooted at $\alpha_{a}$, a $\mathcal{T}\left(-\infty, \alpha_{a}\right)$ and a $\mathcal{T}\left(\alpha_{a+1}, \infty\right)$ rooted at $\alpha_{a+1}$, a $\mathcal{T}\left(-\infty, \alpha_{a+1}\right)$ and a $\mathcal{T}\left(\alpha_{a+2}, \infty\right)$ rooted at $\alpha_{a+2}$, and so on, until we reach 0 , which will have a $\mathcal{T}\left(-\infty, \alpha_{a+b}\right)$ rooted at it. We therefore have $\gamma_{+}\left(A_{i}\right)=\alpha_{a}$ and $\beta_{+}\left(T_{i}\right)=\alpha_{a+b}$.

Similarly, if we start with a tree $U_{j}$ representing $\left(B_{j} 0\right)$, we need to construct $E_{j}=\alpha_{d} \cdots \alpha_{d+e}$. Let $\alpha_{d+e}$ be the smallest vertex with an edge to $0, \alpha_{d+e-1}$ the smallest vertex greater than $\alpha_{d+e}$ with an edge to $\alpha_{d+e}, \alpha_{d+e-2}$ the smallest vertex greater than $\alpha_{d+e-1}$ with an edge to $\alpha_{d+e-1}$, and so on, until we reach a vertex $\alpha_{d}$ such that no $x>\alpha_{d}$ has an edge to $\alpha_{d}$. In this case, $\alpha_{d}>\alpha_{d+1}>\cdots>\alpha_{d+e}$, $\gamma_{-}\left(B_{j}\right)=\alpha_{d}$, and $\beta_{-}\left(U_{j}\right)=\alpha_{d+e}$.

Now that we have constructed the $D_{i}$ 's and $E_{j}$ 's, we can concatenate them into a ring by placing an edge from the last vertex of each $D_{i}$ to the first vertex of $E_{i}$, and the last vertex of $E_{i}$ to the first of $D_{i+1}$. We delete the edges from the last vertices of the $D_{i}$ 's and $E_{i}$ 's to 0 , and leave the trees incident upon the vertices of the $D_{i}$ 's and $E_{i}$ 's in place.

Let $\alpha_{a}$ be the first vertex of $D_{i}$. The inequality $\gamma_{+}\left(A_{i}\right)>\beta_{-}\left(U_{i-1}\right)$ then tells us that $\alpha_{a}$ exceeds the last vertex of $E_{i-1}$, i.e., $\alpha_{a}>\alpha_{a-1}$. Similarly, if $\alpha_{d}$ is the first vertex of $E_{i}$, the inequality $\gamma_{-}\left(B_{i}\right)<\beta_{+}\left(T_{i}\right)$ tells us that $\alpha_{d}<\alpha_{d-1}$. This tells us that the vertices we wish to be upper are indeed upper, and the ones we wish to be lower, lower. The $\mathcal{T}\left(-\infty, \alpha_{a+b}\right)$ of $T_{i}$ rooted at 0 must be unrooted and 
rerooted on the vertices of $E_{i}$ and the first vertex of $D_{i+1}$, so as to represent $A_{i}$ in the form (7); similarly, the $\mathcal{T}\left(\alpha_{d+e}, \infty\right)$ of $U_{j}$ rooted at 0 must be rerooted on the vertices of $D_{j+1}$ and the first vertex of $E_{j+1}$, so that $B_{j}$ is represented in the form (8). From the inequalities we have established, we see that this can be done in a unique way. This construction of a unicyclic representation of $(A)(B)$ shows us that we have a map from the set of things counted by the right-hand side of (5) to the set of things counted by its left-hand side. This map is inverse to our previous map, so both maps are bijections, and the left- and right-hand sides of (6) are equal. This completes the proof.

Corollary 9. If every member of $A$ is less than every member of $B$, then

$$
N_{u}^{\text {uni }}((A),(B))=0
$$

and

$$
N^{\mathrm{uni}}((A)(B))=N_{u}^{\mathrm{uni}}((B),(A))=\sum_{k \geq 1} \frac{1}{k} \bar{N}_{k}((A)) \bar{N}_{k}((B))
$$

where

$$
\bar{N}_{k}((D))=\sum_{\left(D_{1} \cdots D_{k}\right)=(D)} \prod_{i} N^{\prime}\left(\left(D_{i} 0\right)\right) .
$$

Proof. If every member of $A$ is less than every member of $B$, then $N_{\beta_{+}>\gamma_{-}\left(B_{i}\right)}^{\prime}\left(\left(A_{i} 0\right)\right)$ must always vanish, yielding $N_{u}^{\text {uni }}((A),(B))=0$. On the other hand, if $T$ is a tree with root 0 representing $\left(B_{i} 0\right)$, then the condition $\beta_{+}(T)>\gamma_{-}\left(A_{i}\right)$ is vacuous, so

$$
N_{\beta_{+}>\gamma_{-}\left(A_{i}\right)}^{\prime}\left(\left(B_{i} 0\right)\right)=N^{\prime}\left(\left(B_{i} 0\right)\right) \text {. }
$$

Similarly,

$$
N_{\beta_{-}<\gamma_{+}\left(B_{j+1}\right)}^{\prime}\left(\left(A_{j} 0\right)\right)=N^{\prime}\left(\left(A_{j} 0\right)\right)
$$

and substituting into (6) yields the desired result. Of course, the first equation is obvious and does not require Proposition 8 .

\section{Symmetries OF CONCATENATION MULTiPLiCITIES}

If $\pi$ is a permutation on $\{1, \ldots, n\}$ and $\rho$ is a permutation on $\{1, \ldots, m\}$, let the concatenation of $\pi$ and $\rho, \pi \| \rho$, be the permutation on $\{1, \ldots, m+n\}$ that satisfies $(\pi \| \rho)(j)=\pi(j)$ for $j=1, \ldots, n$, and $(\pi \| \rho)(j)=n+\rho(j-n)$ for $j=n+1, \ldots$, $m+n$. Also, let $\sigma_{n}$ be the (self-inverse) permutation on $\{1, \ldots, n\}$ sending each $i$ to $n+1-i$, and let $\bar{\pi}$, the flip of $\pi$, be $\sigma_{n} \pi^{-1} \sigma_{n}$. Since we have

$$
\overline{\left(n a_{n}\right) \cdots\left(2 a_{2}\right)\left(1 a_{1}\right)}=\left(n \sigma_{n}\left(a_{1}\right)\right) \cdots\left(2 \sigma_{n}\left(a_{n-1}\right)\right)\left(1 \sigma_{n}\left(a_{n}\right)\right) \text {, }
$$

there is a natural bijection between the representations of $\pi$ and those of $\bar{\pi}$; therefore,

$$
N(\pi)=N(\bar{\pi}) .
$$

Our formula for $N^{\text {uni }}$ now yields some symmetry properties of the multiplicity of a concatenation.

Theorem 10. $N\left(\rho_{1}\left\|\rho_{2}\right\| \rho_{3} \| \rho_{4}\right)=N\left(\rho_{1}\left\|\rho_{3}\right\| \rho_{2} \| \rho_{4}\right)$, for all (possibly empty) $\rho_{1}, \rho_{2}, \rho_{3}$, and $\rho_{4}$.

Theorem 11. $N\left(\overline{\rho_{1}} \| \rho_{2}\right)=N\left(\rho_{1} \| \rho_{2}\right)$, for all $\rho_{1}$ and $\rho_{2}$. 
It is a corollary of these results that, in a concatenation $\pi_{1}\|\cdots\| \pi_{q}$, the permutations making up the concatenation can have their order rearranged, or be flipped, without affecting the overall multiplicity.

Proof of Theorem 10. To prove Theorem 10, let $\pi=\left(\rho_{1}\left\|\rho_{2}\right\| \rho_{3} \| \rho_{4}\right)$ be decomposed into disjoint cycles, $\pi=\pi_{1} \cdots \pi_{q}$. Then there is a corresponding decomposition $\pi^{\prime}=\left(\rho_{1}\left\|\rho_{3}\right\| \rho_{2} \| \rho_{4}\right)=\pi_{1}^{\prime} \cdots \pi_{q}^{\prime}$, where each $\pi_{i}^{\prime}$ is a conjugate of $\pi_{i}$ by an additive translation. Now since, by Lemma 2,

$$
N(\pi)=\sum_{\chi \text { an involution of }} \prod_{\chi(1, \ldots, q\}} N^{\mathrm{tree}}\left(\pi_{i}\right) \prod_{\chi(i)=j, i<j} N^{\mathrm{uni}}\left(\pi_{i} \pi_{j}\right) .
$$

and similarly for $\pi^{\prime}$, it will do to show that

$$
N^{\text {tree }}\left(\pi_{i}\right)=N^{\text {tree }}\left(\pi_{i}^{\prime}\right) \quad \text { for all } i
$$

and

$$
N^{\mathrm{uni}}\left(\pi_{i} \pi_{j}\right)=N^{\mathrm{uni}}\left(\pi_{i}^{\prime} \pi_{j}^{\prime}\right) \quad \text { for all distinct } i \text { and } j \text {. }
$$

However, $\pi_{i}^{\prime}$ and $\pi_{i}$ are conjugate by an additive translation, and additive translation is order-preserving. This implies (11). As for (12), if $\pi_{i}$ and $\pi_{j}$ come from the same $\rho_{k}, \pi_{i}^{\prime} \pi_{j}^{\prime}$ and $\pi_{i} \pi_{j}$ are conjugate by an additive translation, so the equality follows. Otherwise, every element in the domain of $\pi_{i}$ is smaller than every element in the domain of $\pi_{j}$, or vice-versa, so by (9),

$$
N^{\mathrm{uni}}\left(\pi_{i} \pi_{j}\right)=\sum_{k \geq 1} \frac{1}{k} \bar{N}_{k}\left(\pi_{i}\right) \bar{N}_{k}\left(\pi_{j}\right)
$$

and similarly for $N^{\text {uni }}\left(\pi_{i}^{\prime} \pi_{j}^{\prime}\right)$. Conjugacy by an additive translation then implies that $\bar{N}_{k}\left(\pi_{i}\right)=\bar{N}_{k}\left(\pi_{i}^{\prime}\right)$ for all $i$, proving (12) and Theorem 10 .

Here is an alternate proof of (12) that does not use (9). First, look at a concatenation of two permutations, $\pi$ a permutation on $\{1, \ldots, n\}$ and $\rho$ a permutation on $\{1, \ldots, m\}$. In Lemma 12, below, we give an explicit bijection between the sets of representations of $(\pi \| \rho)$ and $(\rho \| \pi)$. If $\rho$ and $\pi$ are cycles, then, by Lemmas 2 and 12 .

$$
\begin{aligned}
N^{\text {tree }}(\pi) N^{\text {tree }}(\rho)+N^{\text {uni }}(\pi \| \rho) & =N(\pi \| \rho) \\
& =N(\rho \| \pi) \\
& =N^{\text {tree }}(\rho) N^{\text {tree }}(\pi)+N^{\text {uni }}(\rho \| \pi),
\end{aligned}
$$

so $N^{\text {uni }}(\pi \| \rho)=N^{\text {uni }}(\rho \| \pi)$. Together with the independence of $N^{\text {uni }}$ under conjugation by an order-preserving map, this implies (12).

Lemma 12. The two permutations $(\pi \| \rho)$ and $(\rho \| \pi)$ of $\{1, \ldots, m+n\}$ have the same multiplicity $N(\pi \| \rho)=N(\rho \| \pi)$. Moreover, this equality comes from an explicit bijection of representations.

Proof. Represent $(\pi \| \rho)$ as a product of transpositions,

$$
(\pi \| \rho)=\left(m+n a_{m+n}\right) \cdots\left(1 a_{1}\right) .
$$

We will exhibit a sequence $\left(b_{1}, \ldots, b_{m+n}\right)$ with

$$
(\rho \| \pi)=\left(m+n b_{m+n}\right) \cdots\left(1 b_{1}\right) .
$$


Let

$$
\psi(x)= \begin{cases}m+x & \text { if } 1 \leq x \leq n \\ x-n & \text { if } n+1 \leq x \leq m+n\end{cases}
$$

be the permutation of $\{1, \ldots, m+n\}$ that moves the first $n$ terms past the last $m$. Then by definition

$$
(\pi \| \rho)=\pi \cdot \psi^{-1} \rho \psi
$$

and similarly

$$
(\rho \| \pi)=\rho \cdot \psi \pi \psi^{-1}
$$

Using $\psi, 13$ becomes

$$
\pi \cdot \psi^{-1} \rho \psi=\left[\left(m+n a_{m+n}\right) \cdots\left(n+1 a_{n+1}\right)\right]\left[\left(n a_{n}\right) \cdots\left(1 a_{1}\right)\right]
$$

Rearrange terms to get:

$$
\mathrm{id}=\left[\psi \pi^{-1}\left(m+n a_{m+n}\right) \cdots\left(n+1 a_{n+1}\right)\right]\left[( n a _ { n } ) \cdots \left(\begin{array}{ll}
1 & \left.\left.a_{1}\right) \psi^{-1} \rho^{-1}\right] .
\end{array}\right.\right.
$$

Now we use repeatedly the conjugation rule $\tau(x y) \tau^{-1}=(\tau(x) \tau(y))$ to obtain

$$
\mathrm{id}=\left[\left(m b_{m}\right) \cdots\left(\begin{array}{ll}
1 & b_{1}
\end{array}\right) \psi \pi^{-1}\right]\left[\psi^{-1} \rho^{-1}\left(m+n b_{m+n}\right) \cdots\left(m+1 b_{m+1}\right)\right]
$$

where

$$
b_{x}= \begin{cases}\psi \pi^{-1}\left(a_{x+n}\right) & \text { if } 1 \leq x \leq m \\ \rho \psi\left(a_{x-m}\right) & \text { if } m+1 \leq x \leq m+n\end{cases}
$$

Now rearrange again to get

$$
(\rho \| \pi)=\rho \cdot \psi \pi \psi^{-1}=\left(m+n b_{m+n}\right) \cdots\left(1 b_{1}\right) .
$$

This shows us that (14) induces a map from the representations of $(\pi \| \rho)$ to those of $(\rho \| \pi)$. Interchanging the roles of $m$ and $n$, and of $\rho$ and $\pi$, in (14) will give us a map from representations of $(\rho \| \pi)$ to those of $(\pi \| \rho)$, and it will be inverse to this map. This shows that the pairing

$$
\left(a_{1}, \ldots, a_{m+n}\right) \longleftrightarrow\left(b_{1}, \ldots, b_{m+n}\right)
$$

yields a bijection of representations of $(\pi \| \rho)$ and $(\rho \| \pi)$.

Proof of Theorem 11. Let $\rho_{1}$ act on $\{1, \ldots, n\}$ and $\rho_{2}$ act on $\{1, \ldots, m\}$. If we go through the same analysis as in Theorem 10, we will see that it suffices to prove that, for any disjoint cycles $\pi$ and $\mu$ with domain contained in $\{1, \ldots, n\}$, and any cycle $\nu$ with domain contained in $\{n+1, \ldots, m+n\}$, that

$$
\begin{gathered}
N^{\text {tree }}\left(\sigma_{n} \pi^{-1} \sigma_{n}\right)=N^{\text {tree }}(\pi), \\
N^{\text {uni }}\left(\sigma_{n}(\pi \mu)^{-1} \sigma_{n}\right)=N^{\text {uni }}(\pi \mu),
\end{gathered}
$$

and

$$
N^{\mathrm{uni}}\left(\sigma_{n} \pi^{-1} \sigma_{n} \nu\right)=N^{\mathrm{uni}}(\pi \nu) .
$$

Let a permutation $\phi$ have domain $\left\{e_{1}<e_{2}<\cdots<e_{r}\right\} \subseteq\{1, \ldots, n\}$. Now $\sigma_{n}$ is order-reversing on the domain of $\phi$, so there is a natural bijection between the representations of $\phi$ and those of $\sigma_{n} \phi^{-1} \sigma_{n}$, given by sending the representation

$$
\phi=\left(e_{r} e_{a_{r}}\right) \cdots\left(e_{2} e_{a_{2}}\right)\left(e_{1} e_{a_{1}}\right)
$$

to the representation

$$
\sigma_{n} \phi^{-1} \sigma_{n}=\left(\sigma_{n}\left(e_{1}\right) \sigma_{n}\left(e_{a_{1}}\right)\right)\left(\sigma_{n}\left(e_{2}\right) \sigma_{n}\left(e_{a_{2}}\right)\right) \cdots\left(\sigma_{n}\left(e_{r}\right) \sigma_{n}\left(e_{a_{r}}\right)\right) .
$$


Under this bijection, there is an edge from $\sigma_{n}(i)$ to $\sigma_{n}(j)$ in the graph representing $\sigma_{n} \phi^{-1} \sigma_{n}$ just when there is an edge from $i$ to $j$ in the graph representing $\phi$. Hence this bijection preserves treeness or unicyclicity of the representation. Setting $\phi=\pi$ or $\phi=\pi \mu$, this proves $(15)$ and (16). Finally, we need to show (17). If we apply (9) to both of its sides, this reduces to showing that $\bar{N}_{k}\left(\sigma_{n} \pi^{-1} \sigma_{n}\right)=\bar{N}_{k}(\pi)$ for all $k$. Let the reverse of a sequence $A$ be $\tilde{A}$, and write $\sigma_{n}(A)$ to denote the sequence derived by applying $\sigma_{n}$ to each element of $A$. Then, if $\pi=(A), \pi^{-1}$ will be $(\tilde{A})$, and $\sigma_{n} \pi^{-1} \sigma_{n}$ will be $\left(\sigma_{n}(\tilde{A})\right)$, so we wish to show that

$$
\sum_{\left(A_{1} \cdots A_{k}\right)=(A)} \prod_{i} N^{\prime}\left(\left(A_{i} 0\right)\right)=\sum_{\left(A_{1}^{\prime} \cdots A_{k}^{\prime}\right)=\left(\sigma_{n}(\tilde{A})\right)} \prod_{i} N^{\prime}\left(\left(A_{i}^{\prime} 0\right)\right) .
$$

There is a bijection between terms on the left- and right-hand sides of this equation given by setting $A_{i}^{\prime}=\sigma_{n}\left(\tilde{A}_{k+1-i}\right)$. It will, therefore, do to show that

$$
\begin{aligned}
& N^{\prime}\left(\left(\begin{array}{ll}
D & 0
\end{array}\right)\right)=N^{\prime}\left(\left(\sigma_{n}(\tilde{D}) 0\right)\right), \\
& \text { for all sequences } D \text { of distinct elements contained in }\{1, \ldots, n\} .
\end{aligned}
$$

Let the range of $D$ be $\left\{d_{1}<d_{2}<\cdots<d_{q}\right\} . N^{\prime}((D 0))$ is the number of ways of writing $\left(\begin{array}{ll}D & 0)\end{array}\right)$ as an exchange permutation without an exchange $(0 x)$, i.e., the number of ways of representing $\left(\begin{array}{ll}D & 0\end{array}\right)$ as

$$
\left(\begin{array}{ll}
D & 0
\end{array}\right)=\left(d_{q} a_{q}\right) \cdots\left(d_{2} a_{2}\right)\left(d_{1} a_{1}\right), \quad a_{1}, \ldots, a_{q} \in\left\{0, d_{1}, d_{2}, \ldots, d_{q}\right\} .
$$

Extend $\sigma_{n}$ to $\{0,1, \ldots, n\}$ by setting $\sigma_{n}(0)=0$. If we invert both sides of (19) and conjugate it by $\sigma_{n}$, we get

$$
\left(\sigma_{n}(\tilde{D}) 0\right)=\left(\sigma_{n}\left(d_{1}\right) \sigma_{n}\left(a_{1}\right)\right) \cdots\left(\sigma_{n}\left(d_{n-1}\right) \sigma_{n}\left(a_{n-1}\right)\right)\left(\sigma_{n}\left(d_{n}\right) \sigma_{n}\left(a_{n}\right)\right)
$$

Since $\sigma_{n}$ is order-reversing on $\left\{d_{1}, \ldots, d_{q}\right\}$, this gives a bijection between the representations of $\left(\begin{array}{ll}D & 0)\end{array}\right)$ and those of $\left(\sigma_{n}(\tilde{D}) 0\right)$. This proves $(\sqrt{19})$, and hence Theorem 11 .

\section{The most Likely Permutation, For AlL $n$}

After the following two lemmas, we will be able to answer Robbins and Bolker's question by determining, for each $n$, which permutation on $\{1, \ldots, n\}$ has greatest multiplicity.

Given a sequence $D$ of distinct positive integers, let $\bar{N}_{k}^{\prime}((D))$ be $\bar{N}_{k}((D))$ with the restriction that, in the sets of tree representations that this expression counts, we only count tree representations containing a transposition $(d 0)$, where $d$ is the smallest element of $D$.

Lemma 13. Let $k$ be given. For a fixed-length sequence $D$ of distinct positive integers, the quantities

1. $\bar{N}_{k}^{\prime}((D))$,

2. $\bar{N}_{k}((D))-\bar{N}_{k}^{\prime}((D))$, and

3. $\bar{N}_{k}((D))$

are each separately maximized when $D$ is a cyclic permutation of a decreasing sequence. Moreover, for 1 and 3, if $k=1$, this is the only case where the maximum occurs. 
Proof. Without loss of generality, let $D$ end in its smallest element, which we call $d$. We claim that

$$
\bar{N}_{k}((D))-\bar{N}_{k}^{\prime}((D))=\sum_{D^{\prime} D^{\prime \prime}=D} \bar{N}_{k}\left(\left(D^{\prime}\right)\right) N^{\text {tree }, d}\left(\left(D^{\prime \prime}\right)\right)
$$

and

$$
\frac{1}{k} \bar{N}_{k}^{\prime}((D))=\sum_{J D_{1} \cdots D_{k}=D} N^{\prime}((J 0)) N^{\prime}\left(\left(D_{1} 0\right)\right) \cdots N^{\prime}\left(\left(D_{k-1} 0\right)\right) N^{\text {tree }, d}\left(\left(D_{k}\right)\right),
$$

where in these sums $J$, but not $D^{\prime}, D^{\prime \prime}$, or the $D_{i}$ 's, may be empty. The lemma will follow from these claims.

To see where $\bar{N}_{k}^{\prime}((D))$ is maximized, use (21). If $D$ is decreasing, then $J 0$, $D_{1} 0, \ldots, D_{k-1} 0$, and $D_{k}$ will also be decreasing, so by Lemma 1 , each term on the right-hand side of (21) will be maximized. If $k=1$, then the term $N^{\text {tree,d}}((D))$ will appear on the right-hand side of (21). By Lemma when $D$ is a cyclic permutation of a decreasing subsequence, so (21) is maximized only when claimed.

$\bar{N}_{k}((D))$ and $\bar{N}_{k}((D))-\bar{N}_{k}^{\prime}((D))$ must be handled together by induction on the length of $D$. For each possible length of $D$, if $\bar{N}_{k}^{\prime}((D))$ and $\bar{N}_{k}((D))-\bar{N}_{k}^{\prime}((D))$ are maximized when $D$ is decreasing, $\bar{N}_{k}((D))$ obviously will be as well, and, if $k=1$, this will be the only place the maximum occurs, since it is the only place where $\bar{N}_{k}^{\prime}((D))$ is maximized. For $\bar{N}_{k}((D))-\bar{N}_{k}^{\prime}((D))$, use 20). The induction hypothesis and Lemma 1 will then show that the right-hand side of $(20)$ is maximized when $D$ is decreasing.

We now prove our claims.

Proof of (20). To prove (20), look at its left-hand side. It counts $k$-tuples $\left(T_{1}, \ldots, T_{k}\right)$ of trees rooted at 0 , representing $\left(D_{1} 0\right), \ldots,\left(D_{k} 0\right)$, respectively, where $\left(D_{1} \cdots D_{k}\right)=$ $(D)$ and $T_{1}, \ldots, T_{k}$ do not contain the transposition $(d 0)$. The $T_{i}$ 's must contain a unique transposition $(d e)$, where $e \neq d$; if this transposition is in $T_{j}$, removing it from $T_{j}$ will leave us with a tree $U$ rooted at $d$ and a tree $V$ rooted at 0 which jointly represent $\left(D_{j} 0\right)(d e)$. Then $d$ and 0 must appear in separate cycles in $\left(D_{j} 0\right)(d e)$, so $d$ appears after $e$ in $D_{j}$. Recall that the sequence $D$ ends with the element $d$. Let $D^{\prime}$ be the initial segment of $D$ ending in $e$, and let $D^{\prime \prime}$ be the remainder of $D$, so $D=D^{\prime} D^{\prime \prime}$. Since $d$ appears after $e$ in $D_{j}$, it follows that $D^{\prime \prime}$ is a subsequence of $D_{j}$. Delete $D^{\prime \prime}$ from $D_{j}$ to give $D_{j}^{\prime}$. Now

$$
\left(D_{j} 0\right)(d e)=\left(D_{j}^{\prime} 0\right)\left(D^{\prime \prime}\right),
$$

so $U$ represents $\left(D^{\prime \prime}\right)$ and $V$ represents $\left(D_{j}^{\prime} 0\right)$. In fact, $T_{1}, \ldots, T_{j-1}, V, T_{j+1}, \ldots$, $T_{k}$ represent $\left(D_{1} 0\right), \ldots,\left(D_{j-1} 0\right),\left(D_{j}^{\prime} 0\right),\left(D_{j+1} 0\right), \ldots,\left(D_{k} 0\right)$, respectively, so $\left(T_{1}, \ldots, T_{j-1}, V, T_{j+1}, \ldots, T_{k}\right)$ is a representation of the form counted by $\bar{N}_{k}\left(\left(D^{\prime}\right)\right)$. We have already remarked that $U$ is a tree rooted at $d$ representing $D^{\prime \prime}$. This gives a map from the set counted by the left-hand side of (20) to the set counted by the right-hand side of (20). This map is easily seen to be invertible, proving (20).

Proof of (21). The left-hand side of (21) counts the same thing as the left-hand side of (20), except that the transposition $(d 0)$ is required instead of forbidden. Also, we assume that $D_{k}$ contains $d$; this gives the factor of $\frac{1}{k}$. Let $D_{k}=E J$, where $E$ ends with $d$. If the transposition $(d 0)$ is deleted from $T_{k}$, we will be left with a tree $U$ rooted at $d$, representing $(E)$, and a tree $V$ rooted at 0 , representing $(J 0)$. 
However, $J D_{1} \cdots D_{k-1} E=D$, so the $(k+1)$-tuple of trees $\left(V, T_{1}, \ldots, T_{k-1}, U\right)$ is of the form counted by the right-hand side of (21). This gives us a map from the set counted by the left-hand side of (21) to the set counted by the right-hand side of (21). This map is invertible, so (21) holds.

Lemma 14. Among all products of two disjoint cycles of fixed length, $m$ and $p$, acting on a set of cardinality $m+p$, the permutations

$$
\left(\begin{array}{lllll}
c_{1} & c_{2} & \cdots & c_{m}
\end{array}\right)\left(\begin{array}{llll}
c_{m+1} & c_{m+2} & \cdots & c_{m+p}
\end{array}\right)
$$

and

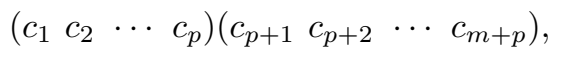

where

$$
c_{1}>c_{2}>\cdots>c_{m+p}
$$

have the highest unicyclic multiplicity. This multiplicity is

$$
N^{\text {uni }}((m+p \cdots p+2 p+1)(p \cdots 21))=\frac{\left(\begin{array}{c}
2 m \\
m
\end{array}\right)\left(\begin{array}{c}
2 p \\
p
\end{array}\right)(m+p+4 m p)-(m+p)\left(\begin{array}{c}
2 m+2 p \\
m+p
\end{array}\right)}{2(m+p)(m+p+1)} .
$$

All other such products have smaller unicyclic multiplicity.

Remark. The proof of the exact formula is left to the reader as a tedious exercise based on (9).

Proof. Let $A=a_{1} \cdots a_{m}$ and $B=b_{1} \cdots b_{p}$ be sequences of distinct positive integers with disjoint ranges, and let $\pi=(A)(B)$. We wish to show that, for fixed $m$ and $p, N^{\mathrm{uni}}(\pi)$ is maximized iff we can write

$$
\begin{aligned}
& (A)=\left(c_{1} \cdots c_{m}\right) \quad \text { and }(B)=\left(d_{1} \cdots d_{p}\right), \text { where } \\
& c_{1}>\cdots>c_{m}, d_{1}>\cdots>d_{p}, \text { and either } c_{m}>d_{1} \text { or } d_{p}>c_{1} .
\end{aligned}
$$

We will induce on $m+p$. Without loss of generality, let 1 be the smallest element in the domain of $\pi$.

Exchange $m$ and $p$, if necessary, to put 1 in $B$, so that we can set $b_{p}=1$. In the case (25), then, we must have $d_{p}=1$, so $c_{1}>\cdots>c_{m}>d_{1}>\cdots>d_{p}$. Let $H$ be a unicyclic representation of $\pi$, with ring $K$. $H$ will contain some transposition $(1 j), j \neq 1$. If 1 is not in $K$, then deleting the edge from 1 to $j$ from $K$ will leave a graph with a tree component $T$ rooted at 1 and a unicyclic component $H^{\prime}$. This graph will represent $\pi(1 j)$. Considering the postman for a moment, we see that in $H$, after stopping for the night at $j$, he must proceed immediately to 1 and traverse $T$. Hence $j$ and 1 are in the same cycle of $\pi$, and

$$
\pi(1 j)=\left(a_{1} \cdots a_{m}\right)\left(b_{1} \cdots b_{q}=j\right)\left(b_{q+1} \cdots b_{p}=1\right),
$$

for some $1 \leq q \leq p-1$. Evidently, $T$ must represent $\left(b_{q+1} \cdots b_{p}=1\right)$, and $H^{\prime}$ must represent $\left(a_{1} \cdots a_{m}\right)\left(b_{1} \cdots b_{q}\right)$. Conversely, given a tree rooted at 1 representing $\left(b_{q+1} \cdots b_{p}\right)$, and a unicycle representing $\left(a_{1} \cdots a_{m}\right)\left(b_{1} \cdots b_{q}\right)$, we can insert an edge from 1 to $b_{q}$ to give a unicyclic representation of $\pi$. Hence

$$
Q(\pi)=\sum_{1 \leq q \leq p-1} N^{\text {tree }, 1}\left(\left(b_{q+1} \cdots b_{p}=1\right)\right) N^{\mathrm{uni}}\left(\left(a_{1} \cdots a_{m}\right)\left(b_{1} \cdots b_{q}\right)\right),
$$


where $Q(\pi)$ is the portion of $N^{\text {uni }}(\pi)$ coming from graphs with 1 not in $K$. By Lemma 1 , the $N^{\text {tree, } 1}$ term will be maximized just when $b_{q+1}>\cdots>b_{p}=1$, which will certainly happen in the case (25), and in this case, by the induction hypothesis, the $N^{\text {uni }}$ term will be maximized as well. Hence $Q(\pi)$ is never any bigger than it is in the case (25).

Let $Q^{\prime}(\pi)=N^{\text {uni }}(\pi)-Q(\pi)$ count the remaining unicyclic representations, that is, those where 1 is in $K$. Let there be edges in $K$ from $z$ to 1 and from 1 to $w$. Since $z>1$ and $w>1,1$ is lower and $w$ is upper, so $(A)$ must be the upper cycle and $(B)$ the lower. Also, using the notation of the proof of Proposition 8, the last element of some $E_{j}$ is 1 ; that is, $1=\beta_{-}\left(U_{j}\right)$ for some $j$. Conversely, if $1=\beta_{-}\left(U_{j}\right)$ for some $j, 1$ will certainly appear in $K$. Therefore, we can think of $Q^{\prime}(\pi)$ as the right-hand side of (6), if we apply the following constraint: in the sets of tree representations that this right-hand side counts, delete all tree representations not containing a tree $U_{j}$ with $1=\beta_{-}\left(U_{j}\right)$.

In the case (25), the inequalities between $\beta_{+}, \gamma_{+}, \beta_{-}$, and $\gamma_{-}$in (6) are vacuous, so we may remove them, and think of $Q^{\prime}(\pi)$ as the right-hand side of (9), subject to our constraint. In all other cases, relaxing these inequalities does not decrease the right-hand side of (6), so it will do to prove that the right-hand side of (9), subject to our constraint, is never bigger than in the case (25).

Our constraint is equivalent to demanding that a transposition $\left(\begin{array}{l}1 \\ 0\end{array}\right)$ occur somewhere in the tree representations of the $B_{j}$ 's, so the right-hand side of (9) with our constraint applied is

$$
\sum_{k \geq 1} \frac{1}{k} \bar{N}_{k}((A)) \bar{N}_{k}^{\prime}((B)) .
$$

It follows from Lemma 13 that (27) is maximized in the case (25). We still need to show exactly when the maximum occurs. For all $D, \bar{N}_{1}((D)) \geq N^{\prime}((D 0))$, and $N^{\prime}\left(\left(\begin{array}{ll}D & 0))\end{array}\right)\right.$ is positive by [5, Theorem 4]. Define $N^{\prime \prime}\left(\left(\begin{array}{ll}D & 0))\end{array}\right)\right.$ to be the number of tree representations of $\left(\begin{array}{ll}D & 0\end{array}\right)$ with 0 as the root and a transposition $(d 0)$ present, for $d$ the smallest element of $D$. Then, for any $D$, let $D^{\prime}=D^{\prime \prime} d$ be $D$ cyclically rotated so that its smallest element, $d$, is at the end. In this case, $\bar{N}_{1}^{\prime}((D)) \geq N^{\prime \prime}\left(\left(D^{\prime \prime} d 0\right)\right)$. As in the proof of Lemma 13, if a tree representation of $\left(D^{\prime \prime} d 0\right)$ rooted at 0 contains the transposition $(d 0)$, we can delete it to get a tree representation of $\left(D^{\prime \prime} d\right)$ rooted at $d$. This transformation is reversible, so

$$
N^{\prime \prime}\left(\left(D^{\prime \prime} d 0\right)\right)=N^{\text {tree }, d}\left(\left(D^{\prime \prime} d\right)\right),
$$

and by [5, Theorem 4], this is positive. Hence $\bar{N}_{1}((D))$ and $\bar{N}_{1}^{\prime}((D))$ are always positive. Also, by Lemma 13, we know that the maximum of $\bar{N}_{1}^{\prime}((D))$ and $\bar{N}_{1}((D))$ can only occur when $D$ is a cyclic permutation of a decreasing sequence. Looking at the $k=1$ term in (27), then, we see that for the maximum to occur, we must be able to write

$$
\begin{aligned}
& (A)=\left(c_{1} \cdots c_{m}\right) \text { and }(B)=\left(d_{1} \cdots d_{p}\right), \text { where } \\
& c_{1}>\cdots>c_{m} \text { and } d_{1}>\cdots>d_{p}=1 .
\end{aligned}
$$

If $p=1$, we are done. Otherwise, write $B=B^{\prime} 1$, and look at the $q=p-1$ term in (26). By the induction hypothesis, if we are at the maximum, (25) must be satisfied for $A$ and $B^{\prime}$. Therefore, either $c_{m}>d_{1}$, and we are done, or $d_{p-1}>c_{1}$.

If $d_{p-1}>c_{1}$, look at the representations of $\pi$ with 1 in $K$. Each $\gamma_{-}\left(B_{j}\right)$ must be exceeded by a $\beta_{+}\left(T_{i}\right)$; therefore, 1 is the only possible value for any $\gamma_{-}\left(B_{j}\right)$, so 
$k=1$ in (6) and $B_{1}=1 B^{\prime}$. Ignoring the other conditions on $\beta_{-}, \gamma_{-}, \beta_{+}$, and $\gamma_{+}$ that occur in (5), we see that

$$
Q^{\prime}((A)(B)) \leq \bar{N}_{1}((A)) N^{\prime \prime}\left(\left(\begin{array}{lll}
B^{\prime} & 0
\end{array}\right)\right) .
$$

Let $A^{\prime}$ denote a shifted version of $A$ with every element greater than every element of $B$. Then, as we know,

$$
Q^{\prime}\left(\left(A^{\prime}\right)(B)\right) \geq \bar{N}_{1}\left(\left(A^{\prime}\right)\right) \bar{N}_{1}^{\prime}((B))=\bar{N}_{1}((A)) \bar{N}_{1}^{\prime}((B)),
$$

and since we know that $\bar{N}_{1}((A))>0$, it will do to show that $\bar{N}_{1}^{\prime}((B))>N^{\prime \prime}\left(\left(\begin{array}{ll}1 & B^{\prime}\end{array}\right)\right)$. Now, $\bar{N}_{1}^{\prime}((B))$ is a sum of $N^{\prime \prime}\left(\left(B_{1} 0\right)\right)$ over cyclic permutations $B_{1}$ of $B$. Since $p>1$, $1 B^{\prime} \neq B^{\prime} 1$, so it will do to show that $N^{\prime \prime}\left(\left(\begin{array}{lll}B^{\prime} & 10 & 0\end{array}\right)>0\right.$; but we know this from above. This shows that the case $d_{p-1}>c_{1}$ cannot occur, completing the proof.

Let $G_{m p}$ denote the right-hand side of (24).

Corollary 15. Fix a conjugacy class of $S_{n}$, and let it consist of all permutations whose representations as products of disjoint cycles consist of an $L_{1}$-cycle, an $L_{2}$ cycle, $\ldots$, and an $L_{q}$-cycle, where $L_{1}+\cdots+L_{q}=n$. Then the permutations of maximum multiplicity in this class are just the permutations where each cycle of length $L$ is of the form $(\Sigma+L \cdots \Sigma+1)$, for some $\Sigma$. Furthermore, the multiplicity of the permutations of maximum multiplicity is

$$
\sum_{\substack{\text { an involution of } \\\{1, \ldots, q\}}} \prod_{\chi(i)=i} C_{L_{i}} \prod_{\chi(i)=j, i<j} G_{L_{i} L_{j}} .
$$

Proof. Let $\pi$ be an arbitrary permutation of $\{1, \ldots, n\}$, and let $\pi=\pi_{1} \cdots \pi_{q}$ be its decomposition into disjoint cycles, where $\pi_{1}$ is an $L_{1}$-cycle, $\pi_{2}$ is an $L_{2}$-cycle, $\ldots$, and $\pi_{q}$ is an $L_{q}$-cycle. By Lemma 2 ,

$$
N(\pi)=\sum_{\chi \text { an involution of }} \prod_{\chi 1, \ldots, q\}} N^{\text {tree }}\left(\pi_{i}\right) \prod_{\chi(i)=j, i<j} N^{\text {uni }}\left(\pi_{i} \pi_{j}\right) .
$$

Using the bounds in Lemmas 4 and 14 then shows that $N(\pi)$ is no bigger than (28) and that the equality condition is as claimed.

Let cycle left be the permutation ( $n n-1 \cdots 21)$, and let double cycle left be the permutation

$$
(n n-1 \cdots m+1)(m \cdots 21)
$$

where $m$ is either $\lfloor n / 2\rfloor$ or $\lceil n / 2\rceil$. Note that the multiplicity of double cycle left is given by Lemma 14 .

Theorem 16. For fixed $n$, the permutations with maximum multiplicity are: for $n=1$, the identity; for $n=2$, cycle left or identity; for $n=3$, cycle left or double cycle left; for $4 \leq n \leq 17$, double cycle left; for $18 \leq n$, the identity.

Proof. For $n \geq 29$, we already know this from Proposition 6, so fix some $n \leq 28$. Let $\left(L_{1}, L_{2}, \ldots, L_{q}\right)$ be a partition of $n$ into positive integers. We can compute (28) for each such partition. The maximum value is found to be achieved for $q=n$ and $L_{1}=\cdots=L_{n}=1$ for $n=1$ and for $18 \leq n \leq 28$; for $q=2$ and $\left\{L_{1}, L_{2}\right\}=\{\lfloor n / 2\rfloor,\lceil n / 2\rceil\}$ for $4 \leq n \leq 17$; and for either $q=1$ and $L_{1}=n$ or $q=2$ and $\left\{L_{1}, L_{2}\right\}=\{1, n-1\}$ for $n=2$ and $n=3$. Applying Corollary 15 completes the proof. 


\section{The LIMITING DistriBUtion OF THE NUMBER OF FIXED POINTS}

Schmidt and Simion [7] consider the exchange permutation (11) where $a_{1}, \ldots, a_{n}$ are picked independently at random from the uniform distribution on $\{1, \ldots, n\}$. They ask what the limiting probability $p_{k}$ of $\pi$ having $k$ fixed points is, as $n$ becomes large.

Theorem 17. If $a_{1}, \ldots, a_{n}$ are picked independently uniformly at random, the limiting probabilities $p_{k}$ of $\pi$ given by (1) having exactly $k$ fixed points as $n \rightarrow \infty$ are given by

where

$$
\sum_{k \geq 0} p_{k} u^{k}=q(u)
$$

$$
q(u)=\exp \left(\frac{1}{2}(u-1)\left(u e^{-2}-6 e^{-1}+4-e^{-2}\right)\right) .
$$

First we recall the Lagrange Inversion Theorem (see [2]), and give three examples that will be used in the proof. For a formal power series $\phi(z)=\phi_{0}+\phi_{1} z+\phi_{2} z^{2}+\cdots$, let $\left[z^{n}\right] \phi$ denote $\phi_{n}$, the coefficient of $z^{n}$.

Theorem 18. (Lagrange Inversion Theorem, or LIT). Let $\phi, \psi$, and $A$ be formal power series with complex coefficients, with $\phi_{0} \neq 0$. If $\psi(z)=z \phi(\psi(z))$, then

$$
\left[z^{n}\right] A(\psi(z))=\frac{1}{n}\left[z^{n-1}\right] \phi^{n}(z) A^{\prime}(z)
$$

for any positive integer $n$.

Here are some applications of LIT that will be used to prove Theorem 17 .

Example 1. The number $\operatorname{Tr}_{n}$ of rooted trees on $n$ labeled vertices. Let $t(z)$ be the exponential generating function for $\operatorname{Tr}_{n}, t(z)=\sum_{n>0} \operatorname{Tr}_{n} z^{n} / n$ !. One checks that $t=z e^{t}$. Apply LIT with $\psi(z)=t(z), \phi(z)=e^{z}$, (note that the constant term of $e^{z}$ is nonzero) and $A(z)=z$ to get $\operatorname{Tr}_{n}=n^{n-1}$.

Example 2. The expansion of $z / t(z)$. Apply LIT with $\psi(z)=t(z), \phi(z)=e^{z}$, and $A(z)=e^{-z}$ to get

$$
\frac{z}{t(z)}=1-\sum_{n>0} \frac{(n-1)^{n-1}}{n !} z^{n}
$$

Example 3. The expansion of $1 /(1-t(z))$. Apply LIT with $\psi(z)=t(z), \phi(z)=e^{z}$, and $A(z)=\frac{1}{1-z}$ to get

$$
\frac{1}{1-t(z)}=\sum_{n \geq 0} \frac{n^{n}}{n !} z^{n}
$$

Proof of Theorem 17. Let $T_{n k}$ be the number of $\left(a_{1}, \ldots, a_{n}\right)$ 's inducing a connected digraph and a permutation with exactly $k$ fixed points. Using [7, Lemma 3.5] gives $\sum_{k>0} T_{1 k}=\sum_{k>0} T_{2 k}=1$, and $\sum_{k>0} T_{n k}=2(n-1)^{n-1}$ for $n \geq 3$. We claim that $T_{11}=T_{22}=1, T_{n 1}=2(n-1)^{n-1}$ for $n \geq 3$, and $T_{n k}$ is zero for all other positive values of $k$. This is because a connected digraph has exactly zero or one fixed points, unless $n=2$, when it must have zero or two. For $t(z)$ as in Example 1, we can then write

$$
T(z, u)=\sum_{n>0, k>0} T_{n k} \frac{z^{n} u^{k}}{n !}=z u+\frac{1}{2} z^{2} u^{2}+u\left(2-\frac{2 z}{t(z)}-2 z-z^{2}\right),
$$


by Example 2. The exponential generating function for the number of ways to pick $\left(a_{1}, \ldots, a_{n}\right)$ overall is

$$
U(z)=\sum_{n \geq 0} \frac{n^{n}}{n !} z^{n}
$$

Observe that $\log U(z)$ is the exponential generating function for the number of connected digraphs on $n$ vertices. The identity $f(z, u)=f(z, 1)$, true for any function $f$ which does not depend on the variable $u$, holds for

$$
f(z, u)=-T(z, u)+\sum_{n>0, k \geq 0} T_{n k} \frac{z^{n} u^{k}}{n !}
$$

However, when $u$ is set equal to 1 , this equals $-T(z, 1)+\log U(z)$, so the exponential generating function for components where $u$ marks the number of fixed points is given by

$$
\sum_{n>0, k \geq 0} T_{n k} \frac{z^{n} u^{k}}{n !}=T(z, u)-T(z, 1)+\log U(z)
$$

Let $p_{n k}$ be the probability of exactly $k$ fixed points occurring in a permutation on $\{1, \ldots, n\}$, and let $q_{n}(u)=\sum_{k \geq 0} p_{n k} u^{k}$. Then

$$
q_{n}(u)=\frac{\left[z^{n}\right] \exp (T(z, u)-T(z, 1)+\log U(z))}{\left[z^{n}\right] U(z)}=\frac{\left[z^{n}\right] U(z) \exp (T(z, u)-T(z, 1))}{\left[z^{n}\right] U(z)} .
$$

By Example 3, $U(z)=1 /(1-t(z))$. Now, since both numerator and denominator of $q_{n}(u)$ are analytic in the complex plane slit along $\left[e^{-1}, \infty\right)$, we can use singularity analysis [3] and the expansion [1] at $z=e^{-1}$

$$
t(z)=1-\sqrt{2(1-e z)}-\frac{1}{3}(1-e z)+O\left((1-e z)^{3 / 2}\right)
$$

to find that $q_{n}(u) \rightarrow q(u)$ pointwise in $u$ as $n \rightarrow \infty$. By [4, Theorem 9.3], the limiting probability $p_{k}$ of exactly $k$ fixed points as $n \rightarrow \infty$ is then given by $\sum_{k \geq 0} p_{k} u^{k}=q(u)$.

Schmidt and Simion [7] also ask what the limiting distribution as $n \rightarrow \infty$ of the number of fixed points is if $a_{1}, \ldots, a_{n}$ are chosen to be a random permutation of $\{1, \ldots, n\}$.

Theorem 19. If $a_{1}, \ldots, a_{n}$ are picked to be a random permutation of $\{1, \ldots, n\}$, the limiting probabilities $\bar{p}_{k}$ of $\pi$ given by (1) having exactly $k$ fixed points as $n \rightarrow \infty$ are given by

$$
\sum_{k \geq 0} \bar{p}_{k} u^{k}=\bar{q}(u)
$$

where

$$
\bar{q}(u)=\exp \left(\frac{1}{2}(u-1)(u+4 e-7)\right) .
$$

Proof. Define $\bar{T}_{n k}, \bar{T}(z, u)$, etc. analogously to the definitions of $T_{n k}, T(z, u)$, etc. in the proof of Theorem 17. Then $\bar{T}_{n k}=0$ for $k>0$ except that $\bar{T}_{11}=1, \bar{T}_{22}=1$, and, for $n \geq 3, \bar{T}_{n 1}=2$ (cf. [7. Theorem 3.13]); therefore,

$$
\bar{T}(z, u)=\sum_{n>0, k>0} \bar{T}_{n k} \frac{z^{n} u^{k}}{k !}=z u+\frac{1}{2} z^{2} u^{2}+2 u\left(e^{z}-1-z-\frac{1}{2} z^{2}\right)
$$


and

$$
\bar{U}(z)=\sum_{n \geq 0} \frac{n !}{n !} z^{n}=\frac{1}{1-z} .
$$

As in Theorem 17,

$$
\bar{q}_{n}(u)=\frac{\left[z^{n}\right] \bar{U}(z) \exp (\bar{T}(z, u)-\bar{T}(z, 1))}{\left[z^{n}\right] \bar{U}(z)} .
$$

Both numerator and denominator are analytic except for a pole at $z=1$, so using singularity analysis [3] there we get $\bar{q}_{n}(u) \rightarrow \bar{q}(u)$ pointwise in $u$ as $n \rightarrow \infty$, and by 4. Theorem 9.3], the generating function of the limiting probabilities is $\bar{q}(u)$. 


\section{REFERENCES}

[1] P. Flajolet and A. M. Odlyzko. Random mapping statistics, in Advances in CryptologyEUROCRYPT '89, Lecture Notes in Computer Science 434, Springer-Verlag, Berlin, 1990, 329-354.

[2] P. Flajolet and R. Sedgewick. The average case analysis of algorithms: counting and generating functions, INRIA research report 1888, April 1993.

[3] P. Flajolet and R. Sedgewick. The average case analysis of algorithms: complex asymptotics and generating functions, INRIA research report 2026, September 1993.

[4] P. Flajolet and R. Sedgewick. The average case analysis of algorithms: multivariate asymptotics and limit distributions, INRIA research report 3162, May 1997.

[5] D. P. Robbins and E. D. Bolker. The bias of three pseudo-random shuffles, Aequationes Mathematicae 22 (1981), 268-292.

[6] H. Robbins. A remark on Stirling's formula, Amer. Math. Monthly 62 (1955), 26-29.

[7] F. Schmidt and R. Simion. Card shuffling and a transformation on $S_{n}$, Aequationes Mathematicae 44 (1992), 11-34. 


\section{FREQUENTLY USED NOTATIONS}

\begin{tabular}{|c|c|}
\hline Notation & Definition \\
\hline$N^{\text {tree }}(\pi)$ & the number of representations of $\pi$ as a tree \\
\hline$N^{\text {uni }}(\pi)$ & the number of representations of $\pi$ as a unicycle \\
\hline$N^{\text {tree, } x}(\pi)$ & $\begin{array}{l}\text { the number of tree representations of } \pi \\
\text { where } a_{x}=x\end{array}$ \\
\hline$N^{\prime}(\pi)$ & $N^{\text {tree }, 0}(\pi)$ \\
\hline$\beta_{+}\left(\right.$resp. $\left.\beta_{-}\right)$ & the largest (resp. smallest) neighbor of 0 \\
\hline$\gamma_{+}\left(\right.$resp. $\left.\gamma_{-}\right)$ & the last (resp. first) element of a sequence \\
\hline$N_{\beta_{+}>x}^{\prime}(\pi)$ & $\begin{array}{l}\text { the number of representations of } \pi \text { as a } \\
\text { tree } T \text { with root } 0 \text { and } \beta_{+}(T)>x\end{array}$ \\
\hline$N_{\beta_{-}<x}^{\prime}(\pi)$ & $\begin{array}{l}\text { the number of representations of } \pi \text { as a } \\
\text { tree } T \text { with root } 0 \text { and } \beta_{-}(T)<x\end{array}$ \\
\hline$N_{u}^{\mathrm{uni}}((A),(B))$ & $\begin{array}{l}\text { the number of unicyclic representations } \\
\text { of the permutation }(A)(B) \text { for which }(A) \\
\text { is the upper cycle }\end{array}$ \\
\hline $\bar{N}_{k}((D))$ & $\sum_{\left(D_{1} \cdots D_{k}\right)=(D)} \prod_{i} N^{\prime}\left(\left(D_{i} 0\right)\right)$ \\
\hline$\pi \| \rho$ & the concatenation of permutations $\pi$ and $\rho$ \\
\hline$\sigma_{n}$ & the permutation sending $i$ to $n+1-i$ \\
\hline $\bar{\pi}$ & $\sigma_{n} \pi^{-1} \sigma_{n}$, if $\pi$ acts on $\{1, \ldots, n\}$ \\
\hline$\tilde{A}$ & the reverse of a sequence $A$ \\
\hline$Q(\pi)$ & $\begin{array}{l}\text { the portion of } N^{\text {uni }}(\pi) \text { coming from graphs } \\
\text { with } 1 \text { not in the ring }\end{array}$ \\
\hline$Q^{\prime}(\pi)$ & $\begin{array}{l}\text { the portion of } N^{\mathrm{uni}}(\pi) \text { coming from graphs } \\
\text { with } 1 \text { in the ring }\end{array}$ \\
\hline $\bar{N}_{k}^{\prime}((D))$ & $\begin{array}{l}\text { the portion of } \bar{N}_{k}((D)) \text { with } a_{d}=0 \\
\text { where } d \text { is the smallest element of } D\end{array}$ \\
\hline$N^{\prime \prime}\left(\left(\begin{array}{ll}D & 0\end{array}\right)\right)$ & $\begin{array}{l}\text { the number of tree representations of }(D 0) \\
\text { with } a_{0}=0 \text { and } a_{d}=0, \text { where } d \text { is the } \\
\text { smallest element of } D\end{array}$ \\
\hline
\end{tabular}

Center for Communications Research 4320 Westerra Court

San Diego, CA 92121

dgoldste@ccrwest.org

dmoews@ccrwest.org 
